\title{
Quadrature Error Functional Expansions for the Simplex When the Integrand Function Has Singularities at Vertices*
}

\author{
By J. N. Lyness and G. Monegato
}

\begin{abstract}
In a previous paper by Lyness, an asymptotic expansion for the quadrature error functional for the $n$-dimensional simplex, valid when the integrand is an analytic function, was given. In this paper we develop the analogue of this result to cover the cases in which the integrand function has singularities of a specified nature at one or more vertices of the simplex.
\end{abstract}

1. Introduction. In Lyness (1978) a particular subdivision $S^{(m)}$ of an $n$-dimensional simplex $S_{n}$ into $m^{n}$ subsimplices of equal $n$-volume is described; and the $m^{n}$-copy quadrature rule $Q^{(m)}\left(S_{n}\right)$ is defined in terms of a set $\{Q\}$ of $n$ ! distinct simplex quadrature rules, one for each type of subsimplex occurring in the subdivision. The principal result of that paper is an asymptotic expansion of the form

$$
Q^{(m)}\left(S_{n}\right) f=\int_{S_{n}} f(\vec{x}) d^{n} x+\sum_{s=1}^{p-1} \frac{B_{s}\left(S_{n} ; Q ; f\right)}{m^{s}}+O\left(m^{-p}\right),
$$

valid when $f(\vec{x})$ and its derivatives of total order $p$ or less are integrable over a region containing the closure of $S_{n}$. This expansion provides a basis for Romberg integration over a simplex.

In this paper we develop the analogue of this result which applies when the integrand function has singularities of a particular nature at one vertex or at several vertices of the simplex $S_{n}$. Our results are based on and bear a strong resemblance to corresponding results reported in Lyness (1976) for integration over a hypercube.

As an example of these results, suppose that the integrand function $f(\vec{x})$ has a singularity at a vertex (the origin) of $S_{n}$ and can be expressed in the form

$$
f(\vec{x})=r^{\alpha} g(\vec{x}), \quad \alpha>-n,
$$

where $g(\vec{x})$ is analytic within the closure of $S_{n}$ in all variables, $\alpha$ is not an integer and

$$
r^{2}=x_{1}^{2}+x_{2}^{2}+\cdots+x_{n}^{2}
$$

Then (1.1) is not valid, but may be replaced by the asymptotic expansion

$$
Q^{(m)}\left(S_{n}\right) f \sim \int_{S_{n}} f(\vec{x}) d^{n} x+\sum_{t=0} \frac{A_{\alpha+n+t}\left(S_{n} ; Q ; f\right)}{m^{\alpha+n+t}}+\sum_{s=1} \frac{B_{s}\left(S_{n} ; Q ; f\right)}{m^{s}} .
$$

Received April 24, 1979.

AMS (MOS) subject classifications (1970). Primary 65D30, 65B15; Secondary 41 A55, 41A60.

* Work performed under the auspices of the U. S. Department of Energy. 
It is also shown that when there are singularities at several vertices, the valid asymptotic expansion is obtained by concatenating the several expansions obtained for the respective cases in which only one singularity is present.

2. Simplex Subdivision. In this section we reintroduce some of the definitions given in Lyness (1978). We use the $n$-dimensional Cartesian coordinate system $\left(x_{1}, x_{2}, \ldots, x_{n}\right)$ and define unit vectors $\vec{i}_{j}, j=1,2, \ldots, n$, along the respective coordinate axes. In terms of these unit vectors, we define a subsidiary set of vectors

$$
\vec{e}_{1}=\vec{i}_{1}, \quad \vec{e}_{j}=\vec{i}_{j}-\vec{i}_{j-1}, \quad j=2,3, \ldots, n .
$$

Clearly,

$$
\overrightarrow{i_{j}}=\vec{e}_{1}+\vec{e}_{2}+\cdots+\vec{e}_{j}
$$

A point of the form

$$
\vec{x}=\left(n_{1} \overrightarrow{i_{1}}+n_{2} \overrightarrow{i_{2}}+\cdots+n_{n} \vec{i}_{n}\right), \quad n_{i}=\text { integer }, i=1,2, \ldots, n,
$$

is termed a grid point. We treat a simplex $S_{n}$, defined by

$$
S_{n}: x_{i}>0, \quad i=1,2, \ldots, n, \quad \sum_{i=1}^{n} x_{i}<1
$$

This is contained in the hypercube $H_{n}$, defined by

$$
H_{n}: 0<x_{i}<1, \quad i=1,2, \ldots, n .
$$

The closure of the simplex and hypercube are denoted by $\bar{S}_{n}$ and $\bar{H}_{n}$, respectively; and $R(D ; \epsilon)$ denotes an $\epsilon$-neighborhood of $D$, i.e. a set which contains all points within a distance $\epsilon$ of some point of $D$.

In what follows, $\left(\mu_{1}, \mu_{2}, \ldots, \mu_{n}\right)=\vec{\mu}_{j}$ stands for one of the $n$ ! permutations of $(1,2, \ldots, n) \equiv \vec{\mu}_{1}$. Let $\vec{a}$ be a grid point, then the simplex $T\left(\vec{a}, \vec{\mu}_{j}\right)$ is one which has vertices $\vec{v}^{(k)}$ at

$$
\vec{v}^{(k)}=\vec{a}+\sum_{i=1}^{k} \vec{e}_{\mu_{i}}, \quad k=0,1, \ldots, n .
$$

We refer to $\vec{\mu}_{j}$ as the orientation index of the simplex $T\left(\vec{a}, \vec{\mu}_{j}\right)$. In Lyness (1978), Section 5, a subdivision of all space was introduced. Let

$$
\Pi_{i, j}(\vec{x})=x_{i}+x_{i+1}+\cdots+x_{j}, \quad 1 \leqslant i \leqslant j \leqslant n ;
$$

then the set of $(n-1)$-dimensional hyperplanes

$$
\Pi_{i, j}(\vec{x})=k_{i, j}, \quad 1 \leqslant i \leqslant j \leqslant n, \forall \text { integer } k_{i, j},
$$

may be employed to form a subdivision of all $n$-dimensional space; that is, every point $\vec{x}$ either lies on one or more of these $(n-1)$-dimensional hyperplanes or lies within a unique simplex $T(\vec{n}, \vec{\mu})$, for some $\vec{n}$ and $\vec{\mu}$, which is bounded by sections of some of these hyperplanes. We refer to this subdivision as $S^{(1)}$ or simply $S$. The subdivision 
$S^{(1)}$ subdivides the hypercube $H_{n}$ into $n$ ! distinct simplices; we denote by $T\left(\vec{\mu}_{j}\right)$ the unique simplex of the form $T\left(\vec{a}, \vec{\mu}_{j}\right)$ which lies within $H_{n}$; in particular, $S_{n}=T\left(\vec{\mu}_{1}\right)$. These $n$ ! simplices are not congruent to one another, but each has $n$-volume $1 / n$ !.

Associated with each orientation index $\vec{\mu}=\left(\mu_{1}, \mu_{2}, \ldots, \mu_{n}\right)$ is an opposite orientation index $\vec{\lambda}=\left(\mu_{n}, \mu_{n-1}, \ldots, \mu_{1}\right)$.

Definition 2.8. A pair of simplices $T(\vec{a}, \vec{\mu})$ and $T(\vec{b}, \vec{\lambda})$ are termed opposite simplices when $\vec{\mu}$ and $\vec{\lambda}$ are opposite orientation indices.

It is straightforward to show that $T(\vec{\mu})$ and $T(\vec{\lambda})$ are inversions of one another about the centroid of $H_{n}$. That is,

$$
\left(x_{1}, x_{2}, \ldots, x_{n}\right) \in T(\vec{\mu}) \Longleftrightarrow\left(1-x_{1}, 1-x_{2}, \ldots, 1-x_{n}\right) \in T(\vec{\lambda}) .
$$

Subsequently, the following characterization of opposite simplices is useful.

Lemma 2.10. Given grid points $\vec{a}, \vec{b}$; given orientation index $\vec{\mu}$, and denoting by $\vec{\lambda}$ its opposite, then

(i) if $\vec{x} \in T(\vec{a}, \vec{\mu})$, there exists a unique point $\vec{y} \in T(\vec{b}, \vec{\lambda})$ such that $\vec{x}+\vec{y}$ is a grid point;

(ii) if $\vec{x} \in T(\vec{a}, \vec{\mu})$ and $\vec{x}+\vec{y}$ is a grid point, then $\vec{y} \in T(\vec{b}, \vec{\lambda})$ for some grid point $\vec{b}$.

The subdivision $S^{(m)}$ is a scaled version of the subdivision $S^{(1)}$ based on $(n-1)$-dimensional hyperplanes

$$
\Pi_{i, j}(\vec{x})=k_{i, j} / m, \quad 1 \leqslant i \leqslant j \leqslant n, \forall \text { integer } k_{i, j} .
$$

This subdivision $S^{(m)}$ may be constructed in two stages. First the hypercube $H_{n}$ is subdivided into $m^{n}$ equal subhypercubes by the $(n-1)$-dimensional hyperplanes

$$
\Pi_{i, i}=x_{i}=\frac{k_{i, i}}{m}, \quad i=1,2, \ldots, n, \forall \text { integer } k_{i, i},
$$

then the subdivision is completed using the hyperplanes

$$
\Pi_{i, j}=\frac{k_{i, j}}{m}, \quad 1 \leqslant i<j \leqslant n, \forall \text { integer } k_{i, j},
$$

-which subdivide each of these subhypercubes into $n$ ! subsimplices in a manner precisely similar to the subdivision of $H_{n}$ using $S^{(1)}$ described above. Thus, the simplex $S_{n}$ is subdivided into $m^{n}$ subsimplices each of which has one of $n$ ! distinct orientation indices. When $m \geqslant n$, there is at least one subsimplex having any specified orientation index within $S_{n}$.

For the purpose of constructing an $m^{n}$-copy rule, Lyness (1978) defined a quadrature rule set for the simplex denoted by

$$
\left\{Q\left(\vec{\mu}_{j}\right), j=1,2, \ldots, n !\right\} .
$$

This consists of a set of $n$ ! distinct quadrature rules, each component rule $Q\left(\vec{\mu}_{j}\right)$ being exact for integrating the constant function over $T\left(\vec{\mu}_{j}\right)$. 
The $m^{n}$-copy of this quadrature rule set is another quadrature rule set denoted by $\left\{Q^{(m)}\left(\vec{\mu}_{j}\right), j=1,2, \ldots, n !\right\}$. The component rule $Q^{(m)}\left(\vec{\mu}_{j}\right)$ is the one obtained by employing the properly scaled version of $Q\left(\vec{\mu}_{k}\right)$ on each subsimplex of $T\left(\vec{\mu}_{j}\right)$ which has orientation index $\vec{\mu}_{k}$, for $k=1,2, \ldots, n !$.

Definition 2.13. A quadrature rule set has polynomial degree $d$ if each component $Q\left(\vec{\mu}_{j}\right)$ has this degree with respect to integration over $T\left(\vec{\mu}_{j}\right)$. In this case it follows that the $m^{n}$-copy of the quadrature rule set is also of this polynomial degree.

Definition 2.14. The quadrature rule set (2.12) is symmetric if each pair of component rules $Q(\vec{\mu})$ and $Q(\vec{\lambda})$, where $\vec{\mu}$ is opposite to $\vec{\lambda}$, satisfies

$$
Q(\vec{\mu}) f=Q(\vec{\lambda}) g
$$

for all $g\left(x_{1}, x_{2}, \ldots, x_{n}\right)=f\left(1-x_{1}, 1-x_{2}, \ldots, 1-x_{n}\right)$.

In terms of weights and abscissas, a symmetric quadrature rule set assigns identical weights to any two abscissas $\vec{x}_{i}$ and $\vec{x}_{j}$ whose midpoint $\left(\vec{x}_{i}+\vec{x}_{j}\right) / 2$ is the centroid of $H_{n}$, these abscissas being associated with simplices $T(\vec{\mu})$ and $T(\vec{\lambda})$ which are linear inversions of one another through the centroid. Associated with a quadrature rule set for a simplex $S_{n}$ is a quadrature rule $Q\left(H_{n}\right)$ for the hypercube $H_{n}$, defined by

$$
Q\left(H_{n}\right) f=\sum_{j=1}^{n !} Q\left(\vec{\mu}_{j}\right) f
$$

From the definitions given above, it follows that

$$
Q^{(m)}\left(H_{n}\right) f=\sum_{j=1}^{n !} Q^{(m)}\left(\vec{\mu}_{j}\right) f .
$$

Furthermore, if the quadrature set (2.12) is of degree $d$ or is symmetric, so also is the associated rule $Q\left(H_{n}\right)$.

In this paper we shall deal extensively with asymptotic expansions of form (1.1) above. These will involve coefficients $B_{s}(D ; Q ; f)$ where $s$ is a positive integer, $f$ is a given function and $Q$ is either a quadrature rule set (when $D$ is a simplex) or a quadrature rule when $D$ is a hypercube.

Definition 2.17 (The SSP conditions). The entities $B_{s}(D ; Q ; f)$ satisfy the standard symmetry and polynomial degree conditions if

(a) When $Q$ is symmetric

$$
B_{s}(D ; Q ; f)=0 \quad \forall s \text { odd. }
$$

(b) When $Q$ is of polynomial degree $d(Q)$

$$
B_{s}(D ; Q ; f)=0, \quad s=1,2, \ldots, d(Q) .
$$

The definitions given in this section are taken directly from Lyness (1978) or are trivial and compatible extensions of those given there.

3. Subdivision Preserving Transformations. In Section 4 we shall derive the analogue of $(1.1)$ for an integration domain $T\left(\vec{\mu}_{j}\right)$ by means of an affine transformation 
of the coordinate system. In this section we discuss a subclass of such affine transformations termed subdivision preserving transformations. These will be used also in Section 5 .

A general affine transformation of the coordinate system has the form

$$
\overline{\vec{x}}=A \vec{x}+\vec{b},
$$

where $A$ is an $n \times n$ nonsingular matrix and $\vec{b}$ is an $n$-vector. A grid-preserving transformation is one of form $(3.1)$ which induces a $(1,1)$ correspondence between grid points in the two coordinate systems. Clearly, the condition for (3.1) to be a grid-preserving transformation is:

Each element of $A, A^{-1}$ and $\vec{b}$ is an integer.

In general, there is no problem in finding a grid-preserving transformation which takes an individual simplex $T(\vec{a}, \vec{\mu})$ into another specified simplex $T\left(\vec{a}^{\prime}, \vec{\mu}^{\prime}\right)$. Once the corresponding vertices have been assigned one obtains $n+1$ linear equations for $\vec{b}$ and the rows of $A$ which can be solved. However, when $n>2$, it is generally unlikely that this transformation will transform other simplices $T(\vec{a}, \vec{\lambda})$ into simplices which are members of the subdivision $S$.

We shall be interested in a subclass of grid-preserving transformations which we now define.

Definition 3.3. An S-preserving affine transformation is one for which the set of hyperplanes

$$
\Pi_{i, j}(\vec{x})=k_{i, j}, \quad 1 \leqslant i \leqslant j \leqslant n, \quad \forall k_{i, j} \text { integer, }
$$

and the set

$$
\Pi_{i, j}(A \vec{x}+\vec{b})=l_{i, j}, \quad 1 \leqslant i \leqslant j \leqslant n, \forall l_{i, j} \text { integer, }
$$

coincide.

It is selfevident that such a transformation also preserves the subdivision $S^{(m)}$ for all positive integer $m$. That it is a grid-preserving transformation follows directly from the circumstance that the grid points are characterized uniquely as the points of intersection of $n(n+1) / 2$ hyperplanes. An $S$-preserving affine transformation induces an automorphism between the orientation indices $\vec{\mu}_{j}(j=1,2, \ldots, n !)$ of the subdivision. This follows since, given $\vec{a}$ and $\vec{\mu}_{j}$ which define the simplex $T\left(\vec{a}, \vec{\mu}_{j}\right)$, there exists a unique simplex say $T(\vec{c}, \overrightarrow{\vec{\mu}})$ such that

$$
\vec{x} \in T\left(\vec{a}, \vec{\mu}_{j}\right) \Longleftrightarrow \overline{\vec{x}} \in T\left(\vec{c}, \overline{\vec{\mu}}_{j}\right) .
$$

Moreover, $\vec{\mu}_{j}$ depends only on $\vec{\mu}_{j}$ and not on $\vec{a}$. Since the inverse transformation exists and is also $S$-preserving, it follows that $(3.4)$ defines a $(1,1)$ correspondence between $\vec{\mu}_{j}$ and $\vec{\mu}_{j}$.

LEMMA 3.5. For a given S-preserving transformation, when $\vec{\lambda}$ and $\vec{\mu}$ are opposite orientation indices, so are $\overrightarrow{\vec{\lambda}}$ and $\overrightarrow{\vec{\mu}}$ (defined by (3.4)). 
This follows from Lemma 2.10. In view of part (i) of this lemma when $\vec{x} \in$ $T(\vec{a}, \vec{\mu})$, there exists a point $\vec{y} \in T(\vec{c}, \vec{\lambda})$ for which $\vec{x}+\vec{y}$ is a grid point. We denote this grid point by $\vec{n}$. Applying the transformation gives

$$
\overline{\vec{x}}+\overline{\vec{y}}=A(\vec{x}+\vec{y})+2 \vec{b}=A \vec{n}+2 \vec{b}
$$

and since the elements of $A$ and of $\vec{b}$ are integers, we see that $\overline{\vec{x}}+\overline{\vec{y}}$ is a grid point. Then application of part (ii) of Lemma 2.10 shows that $\overrightarrow{\vec{x}}$ and $\overrightarrow{\vec{y}}$ are points in opposite simplices, i.e. $\overrightarrow{\vec{\mu}}$ and $\overrightarrow{\vec{\lambda}}$ are opposite orientation indices.

An $S$-preserving transformation may be used to define a new quadrature rule set $\left\{\bar{Q}\left(\vec{\mu}_{j}\right), j=1,2, \ldots, n !\right\}$ in terms of a given quadrature rule set $\left\{Q\left(\vec{\mu}_{j}\right), j=1,2\right.$, $\ldots, n !\}$. Specifically, one defines first the function $\bar{f}$ by

$$
\bar{f}(\overline{\vec{x}})=\bar{f}(A \vec{x}+\vec{b}) \equiv f(\vec{x})
$$

and sets

$$
I\left(\vec{\mu}_{j}\right) f=\int_{T\left(\vec{\mu}_{j}\right)} f(\vec{x}) d^{n} x
$$

and

$$
Q\left(\vec{\mu}_{j}\right) f=\sum_{i=1}^{\nu} w_{i} f\left(\vec{x}^{(i)}\right) .
$$

If the transformation takes $T\left(\vec{\mu}_{j}\right)$ into $T\left(\vec{a}_{j}, \overrightarrow{\vec{\mu}}_{j}\right)$ by a simple change of variable, we find that

$$
I\left(\vec{\mu}_{j}\right) f=I\left(\vec{a}_{j}, \overline{\vec{\mu}}_{j}\right) \bar{f}
$$

and

$$
Q\left(\vec{\mu}_{j}\right) f=\sum_{i=1}^{\nu} w_{i} \bar{f}\left(\overline{\vec{x}}^{(i)}\right) \equiv \bar{Q}\left(\vec{a}_{j}, \overline{\vec{\mu}}_{j}\right) \bar{f}
$$

this relation defining a rule element $\bar{Q}\left(\vec{a}_{j}, \overrightarrow{\vec{\mu}}_{j}\right)$. The rule $\bar{Q}\left(\overline{\vec{\mu}}_{j}\right)$ is simply a translated version of this. Since the set $\overrightarrow{\vec{\mu}}_{j}$ is simply a reordering of the set $\vec{\mu}_{j}$, this procedure may be used to define each element of a set of quadrature rules $\bar{Q}\left(\vec{\mu}_{j}\right), j=1,2, \ldots, n$. Recalling the (geometric) definition of an $m$-copy rule, from (3.8) it also follows

$$
Q^{(m)}\left(\vec{\mu}_{j}\right) f=\bar{Q}^{(m)}\left(\vec{a}_{j}, \overline{\vec{\mu}}_{j}\right) \bar{f}
$$

The following theorem is required for establishing the first major result of Section 4.

THEOREM 3.10. Given any orientation index $\vec{\mu}$, there exists an S-preserving affine transformation which transforms $T(\vec{\mu})$ into $S_{n}=T\left(\vec{\mu}_{1}\right)$.

This is a corollary of Lemma 3.11 below.

LEMMA 3.11. Let $\vec{\mu}=\left(\mu_{1}, \mu_{2}, \ldots, \mu_{n}\right)$. The transformation defined by

$$
\begin{aligned}
& \bar{x}_{j}=\Pi_{\mu_{j}, n}(\vec{x})-\Pi_{\mu_{j+1}, n}(\vec{x}), \quad j=1,2, \ldots, n-1, \\
& \bar{x}_{n}=\Pi_{\mu_{n}, n}(\vec{x})
\end{aligned}
$$


(a) is an S-preserving transformation,

(b) transforms the simplex $T(\overrightarrow{0}, \vec{\mu})$ into the simplex $S_{n}=T\left(\overrightarrow{0}, \vec{\mu}_{1}\right)$.

The proof is based on straightforward but tedious manipulation. First, we show from (3.11) that

$$
\begin{aligned}
\Pi_{l, m}(\overline{\vec{x}}) & =\Pi_{\mu_{l}, \mu_{m+1}-1}(\vec{x}) & & \text { when } \mu_{m+1}>\mu_{l}, 1 \leqslant l \leqslant m<n, \\
& =-\Pi_{\mu_{m+1}, \mu_{l}-1}(\vec{x}) & & \text { when } \mu_{m+1}<\mu_{l}, 1 \leqslant l \leqslant m<n,
\end{aligned}
$$

and

$$
\Pi_{l, n}(\overline{\vec{x}})=\Pi_{\mu_{l}, n}(\vec{x}), \quad \forall 1 \leqslant l \leqslant n,
$$

which in view of Definition 3.3, establishes part (a) of the lemma. Part (b) is established by showing in turn that each vertex $\vec{v}^{(k)}$ of $T(0, \vec{\mu})$ is transformed to $\overline{\vec{v}}^{(k)}=$ $\overrightarrow{i_{k}}$, a vertex of $S_{n}$. To do this we note that

where

$$
\vec{v}(k)=\vec{e}_{\mu_{1}}+\vec{e}_{\mu_{2}}+\cdots+\vec{e}_{\mu_{k}}
$$

$$
\vec{e}_{\mu_{j}}=\vec{i}_{\mu_{j}}-\vec{i}_{\mu_{j}-1}, \quad \mu_{j} \neq 1
$$

Since $\vec{e}_{\mu_{j}}$ has at most two nonzero coordinates, these being $x_{\mu_{j}}=+1$ and when $\mu_{j} \neq 1$, $x_{\mu_{j-1}}=-1$, it follows that

$$
\begin{array}{rlrl}
\Pi_{\mu_{k}, n}\left(\vec{e}_{\mu_{j}}\right) & =1, & k=j, \\
& =0, & & \text { otherwise }
\end{array}
$$

giving

$$
\begin{aligned}
\Pi_{\mu_{j}, n}\left(\vec{v}^{(k)}\right)=\sum_{l=1}^{k} \Pi_{\mu_{j}, n}\left(\vec{e}_{\mu}\right) & =1, \quad j=1,2, \ldots, k, \\
& =0, \quad j=k+1, \ldots, n .
\end{aligned}
$$

Thus, when $j \neq n$, we find

$$
\begin{aligned}
\overline{\vec{v}}_{j}^{(k)}=\Pi_{\mu_{j}, n}\left(\vec{v}^{(k)}\right)-\Pi_{\mu_{j+1}, n}\left(\vec{v}^{(k)}\right) & =1, & & k=j, \\
& =0, & & \text { otherwise; }
\end{aligned}
$$

and it is readily verified that the same result holds when $j=n$. This identifies $\overline{\vec{v}}^{(k)}$ with $\vec{i}_{k}$ establishing part (b) of the lemma.

The validity of Theorem 3.10 is now evident since there is a trivial $S$-preserving transformation taking $T(\vec{\mu})$ into $T(\overrightarrow{0}, \vec{\mu})$ in cases in which they are different.

4. Expansion for the Error Functional. The following theorem is a minor generalization of the principal theorem of Lyness (1978).

THEOREM 4.1. For all quadrature sets $\left\{Q\left(\vec{\mu}_{j}\right), j=1,2, \ldots, n !\right\}$ and for all functions $f(\vec{x})$ whose partial derivatives of total order $d+n-1$ or less exist within 
an $\epsilon$ neighborhood of $T\left(\vec{\mu}_{k}\right)$ for some $\epsilon>0$

$$
Q^{(m)}\left(\vec{\mu}_{k}\right) f=I\left(\vec{\mu}_{k}\right) f+\sum_{s=1}^{d+n-1} \frac{B_{s}\left(\vec{\mu}_{k} ; Q ; f\right)}{m^{s}}+O\left(m^{-(d+1)}\right),
$$

where the coefficients $B_{s}$ satisfy the SSP conditions. Moreover, when $f(\vec{x})$ is a polynomial of degree $d$, the term in (4.1) denoted by $O\left(m^{-(d+1)}\right)$ is zero.

Proof. In the case in which $f(\vec{x})$ is an analytic function and $k=1$, i.e., $T\left(\vec{\mu}_{k}\right)$ $=S_{n}$, this theorem is a restatement of Theorems 5.25, 6.2 and 6.6 of Lyness (1978). Examination of the proof of Theorem 5.25 shows that the proof given there stands as long as $f(\vec{x})$ has partial derivatives of total order $d+n-1$ or less within an $\epsilon$-neighborhood of $S_{n}$. This establishes the validity of the theorem when $k=1$. We use this in extending the proof to cover other values of $k$.

The steps of the proof are these. We consider the transformation of Theorem 3.10 with $\vec{\mu}=\vec{\mu}_{k}$ and apply it to some of the results in Section 3. This transformation can be used to define $\bar{f}$ in terms of $f$ by (3.6). It takes $T\left(\vec{\mu}_{k}\right)$ into $T\left(\vec{\mu}_{1}\right)$. Thus, in terms of the notation defined in (3.4)

$$
\overline{\vec{\mu}}_{k}=\vec{\mu}_{1} \text {, }
$$

and (3.7) and (3.9) with $j=k$ take the form

$$
I\left(\vec{\mu}_{k}\right) f=I\left(\vec{\mu}_{1}\right) \bar{f} ; \quad Q^{(m)}\left(\vec{\mu}_{k}\right) f=\bar{Q}^{(m)}\left(\vec{\mu}_{1}\right) \bar{f} .
$$

We have already established (4.1) with $k=1$. This is valid for a general quadrature rule set and any function satisfying the conditions stated in the theorem. Applying (4.1) with $k=1$ to the rule set $\left\{\bar{Q}\left(\vec{\mu}_{j}\right), j=1,2, \ldots, n !\right\}$ and function $\bar{f}(x)$ gives

$$
\bar{Q}^{(m)}\left(\vec{\mu}_{1}\right) \bar{f}-I\left(\vec{\mu}_{1}\right) \bar{f}=\sum_{s=1}^{d+n-1} \frac{B_{s}\left(\vec{\mu}_{1} ; \bar{Q}, \bar{f}\right)}{m^{s}}+O\left(m^{-(d+1)}\right) .
$$

Substituting relations (4.3) into this gives (4.1) as written, the coefficients on the right being defined by

$$
B_{s}\left(\vec{\mu}_{k} ; Q, f\right)=B_{s}\left(\vec{\mu}_{1} ; \bar{Q}, \bar{f}\right) .
$$

The final part of the theorem, which states that the coefficient on the right of (4.1) satisfy the SSP conditions, follows simply from (4.5). Clearly, the polynomial degrees of $\bar{Q}$ and $\bar{f}$ coincide with those of $Q$ and $f$, respectively. And in view of Lemma 3.5, the circumstance that $Q$ is symmetric implies that $\bar{Q}$ is symmetric.

We now proceed to derive an expansion similar to (4.1) but valid when the integrand function $f(\vec{x})$ has a singularity of a certain type at a vertex of the simplex. The derivation depends heavily on corresponding results for integration over a hypercube which are given in Lyness (1976).

Definition 4.6. A function $f(\vec{x})$ is homogeneous of degree $\gamma$ with respect to a point $\vec{p}$ when

$$
f(\lambda(\vec{x}-\vec{p}))=\lambda^{\gamma} f(\vec{x}-\vec{p}), \quad \forall \vec{x} \neq \vec{p} \text { and real } \lambda \neq 0
$$


Note that if such a function has a singularity at $\vec{x}_{s}$, then it has singularities at all points $\alpha \vec{x}_{s}+(1-\alpha) \vec{p}$ for all real $\alpha$.

Definition 4.7. The set $H_{\gamma}^{+}$includes all homogeneous functions of degree $\gamma$ about the origin which have no singularity in the principal quadrant $x_{i} \geqslant 0, i=1,2, \ldots, n$, other than the one at the origin.

THEOREM 4.8 (LyNESS (1976), THEOREM 4.17). Let $f_{\gamma}(\vec{x}) \in H_{\gamma}^{+}$, and let $Q^{(m)}\left(H_{n}\right)$ be the $m^{n}$-copy of $Q\left(H_{n}\right)$, a quadrature rule for the hypercube $H_{n}$. Then

$$
Q^{(m)}\left(H_{n}\right) f_{\gamma}-I\left(H_{n}\right) f_{\gamma} \sim \frac{A_{n+\gamma}\left(H_{n} ; Q ; f_{\gamma}\right)}{m^{n+\gamma}}+\frac{C_{n+\gamma}\left(H_{n} ; Q ; f_{\gamma}\right)}{m^{n+\gamma}} \ln m
$$

$$
+\sum_{s=1} \frac{B_{s}\left(H_{n} ; Q ; f_{\gamma}\right)}{m^{s}}
$$

and $B_{s}\left(H_{n} ; Q ; f_{\gamma}\right)$ and $C_{n+\gamma}\left(H_{n} ; Q ; f_{\gamma}\right)$ satisfy the SSP conditions (see Definition 2.17).

Incidentally, there are many circumstances in which some of these coefficients are zero. Most important is

$$
C_{n+\gamma}=0 \quad \text { unless } \gamma=\text { integer. }
$$

This theorem is also valid when $Q^{(m)}$ is replaced by $\bar{Q}^{[m]}$, which is identical with $Q^{(m)}$ except that the function value at the origin is omitted.

Corresponding results for the simplex $S_{n}$ are given by the following:

THEOREM 4.10. Let $f_{\gamma} \in H_{\gamma}^{+}$. For all quadrature rule sets $\left\{Q\left(\vec{\mu}_{j}\right), j=1,2\right.$, $\ldots, n !\}$

$$
\begin{aligned}
Q^{(m)}\left(S_{n}\right) f_{\gamma}-I\left(S_{n}\right) f_{\gamma} \sim & \frac{A_{n+\gamma}\left(S_{n} ; Q ; f_{\gamma}\right)}{m^{n+\gamma}}+\frac{C_{n+\gamma}\left(S_{n} ; Q ; f_{\gamma}\right)}{m^{n+\gamma}} \ln m \\
& +\sum_{s=1} \frac{B_{s}\left(S_{n} ; Q ; f_{\gamma}\right)}{m^{s}},
\end{aligned}
$$

where the coefficients $B_{s}\left(S_{n} ; Q ; f_{\gamma}\right)$ and $C_{n+\gamma}\left(S_{n} ; Q ; f_{\gamma}\right)$ satisfy the SSP conditions and $C_{n+\gamma}=0$ unless $\gamma$ is an integer.

Proof. We recall from $(2.15)$ that

$$
Q\left(H_{n}\right)=Q\left(S_{n}\right)+\sum_{k=2}^{n !} Q\left(T\left(\vec{\mu}_{k}\right)\right)
$$

from which it follows that

$$
Q^{(m)}\left(S_{n}\right) f_{\gamma}=Q^{(m)}\left(H_{n}\right) f_{\gamma}-\sum_{k=2}^{n !} Q^{(m)}\left(T\left(\vec{\mu}_{k}\right)\right) f_{\gamma}
$$

Since $f_{\gamma}(\vec{x}) \in H_{\gamma}^{+}$, it is analytic in $T\left(\mu_{k}\right), k=2,3, \ldots, n$ !. Thus, we may expand each term in the summation over $\mathrm{k}$ using (4.1). We may expand the first term on the 
right-hand side using (4.8). Doing this gives (4.10) as written, the coefficients being given by

$$
\begin{aligned}
& A_{n+\gamma}\left(S_{n} ; Q ; f_{\gamma}\right)=A_{n+\gamma}\left(H_{n} ; Q, f_{\gamma}\right), \\
& C_{n+\gamma}\left(S_{n} ; Q ; f_{\gamma}\right)=C_{n+\gamma}\left(H_{n} ; Q ; f_{\gamma}\right), \\
& B_{s}\left(S_{n} ; Q, f_{\gamma}\right)=B_{s}\left(H_{n} ; Q ; f_{\gamma}\right)-\sum_{k=2}^{n !} B_{s}\left(T\left(\vec{\mu}_{k}\right) ; Q ; f_{\gamma}\right) .
\end{aligned}
$$

Since each term on the right-hand side of the third relation individually satisfies the SSP conditions, so does $B_{s}\left(S_{n} ; Q ; f_{\gamma}\right)$. The same result holds for $C_{n+\gamma}\left(S_{n} ; Q ; f_{\gamma}\right)$, which furthermore is zero unless $\gamma$ is an integer.

We may now apply the previous theorems to prove the following:

THEOREM 4.14. Let

$$
f(\vec{x})=\sum_{i=0}^{d-1} f_{\alpha+i}(\vec{x})+g(\vec{x}),
$$

where $f_{\alpha+i} \in H_{\alpha+i}^{+}$and $g(\vec{x})$ is a function all of whose partial derivatives of total order $l$ or less are integrable over an $\epsilon$-region of $S_{n}$. Then, for all quadrature rule sets $\left\{Q\left(\vec{\mu}_{j}\right), j=1,2, \ldots, n !\right\}$

$$
\begin{aligned}
Q^{(m)}\left(S_{n}\right) f-I\left(S_{n}\right) f= & \sum_{i=0}^{d-1} \frac{A_{n+\alpha+i}\left(S_{n} ; Q ; f\right)}{m^{n+\alpha+i}}+\frac{C_{n+\alpha+i}\left(S_{n} ; Q ; f\right)}{m^{n+\alpha+i}} \ln m \\
& +\sum_{s=1}^{l-1} \frac{B_{s}\left(S_{n} ; Q ; f\right)}{m^{s}}+O\left(m^{-l}\right),
\end{aligned}
$$

where $B_{s}\left(S_{n} ; Q ; f\right)$ and $C_{n+\alpha+i}\left(S_{n} ; Q ; f\right)$ satisfy the SSP conditions, and $C_{n+\alpha+i}=0$ unless $\alpha=$ integer.

Proof. Since $Q^{(m)}\left(S_{n}\right)$ is a linear operator,

$$
Q^{(m)}\left(S_{n}\right) f=\sum_{i=0}^{d-1} Q^{(m)}\left(S_{n}\right) f_{\alpha+i}+Q^{(m)}\left(S_{n}\right) g .
$$

Replacing the terms on the right of (4.16) by the expansions given in Theorems 4.10 and 4.1 with $k=1$ gives (4.15) directly with

$$
\begin{aligned}
& A_{n+\alpha+i}\left(S_{n} ; Q ; f\right)=A_{n+\alpha+i}\left(S_{n} ; Q ; f_{\alpha+i}\right), \\
& C_{n+\alpha+i}\left(S_{n} ; Q ; f\right)=C_{n+\alpha+i}\left(S_{n} ; Q ; f_{\alpha+i}\right), \\
& B_{s}\left(S_{n} ; Q ; f\right)=B_{s}\left(S_{n} ; Q ; g\right)+\sum_{i=0}^{d-1} B_{s}\left(S_{n} ; Q ; f_{\alpha+i}\right) .
\end{aligned}
$$

This theorem establishes expansion (4.15) for a wide class of integrand functions having a singularity of the origin. For example, let

$$
f(\vec{x})=r^{\alpha} h(r) g\left(x_{1}, x_{2}, \ldots, x_{n}\right),
$$


where

$$
r=|\vec{x}|=\left(x_{1}^{2}+x_{2}^{2}+\cdots+x_{n}^{2}\right)^{1 / 2}
$$

and $h(r)$ and $g\left(x_{1}, x_{2}, \ldots, x_{n}\right)$ are analytic functions of their stated variables. This function may be expressed, using multivariate Taylor expansion, in form (4.14) with any value of $d$ and so (4.15) applies as written to this function.

A more thorough description of the classes of functions to which this and similar theorems may be applied is given in Lyness (1976).

5. Singularities at Other Vertices. The result in the previous section gives the error functional expansion for the case in which the integrand function has a singularity at one specified vertex of $S_{n}$, namely the origin. In this section we first extend the theory to establish the corresponding result when the singularity is at some other vertex.

An extension of this sort for the corresponding result for the hypercube is trivial to establish. For example, if the singularity is at $\vec{i}_{1}=(1,0,0, \ldots, 0)$, one may simply apply coordinate transformation

$$
x_{1}^{\prime}=1-x_{1}, \quad x_{j}^{\prime}=x_{j}, \quad j=2,3, \ldots, n .
$$

This takes the hypercube into itself, the hypercube subdivision into itself, moves the singularity to the required point, and alters the quadrature rule in an obvious manner.

For the simplex, one can proceed in this way only if the transformation is S-preserving. It turns out that the simplest transformation, one which interchanges two vertices leaving the others unaltered, is not $S$-preserving.

THEOREM 5.2. The transformation

$$
\begin{aligned}
& \bar{x}_{1}=1-\Pi_{1, n}(\vec{x}), \\
& \bar{x}_{j}=x_{j-1}, \quad j=2,3, \ldots, n,
\end{aligned}
$$

(1) transforms the origin to the point $\vec{i}_{1}$, transforms $\vec{i}_{n}$ to the origin and transforms $\vec{i}_{j}$ to $\vec{i}_{j+1}$ for $j=1,2, \ldots, n-1$;

(2) transforms $S_{n}$ into itself;

(3) is an S-preserving transformation.

Proof. It is trivial to verify item (1); and item (2) follows from item (1) immediately.

To establish that the transformation is S-preserving, one need verify only that

$$
\begin{aligned}
& \Pi_{1, l}(\overrightarrow{\vec{x}})=1-\Pi_{1, n}(\vec{x})+\sum_{j=2}^{l} x_{j-1}=1-\Pi_{l, n}(\vec{x}), \quad l=2,3, \ldots, n, \\
& \Pi_{k, l}(\overrightarrow{\vec{x}})=\sum_{j=k}^{l} \bar{x}_{j}=\sum_{j=k}^{l} x_{j-1}=\sum_{j=k-1}^{l-1} x_{j}=\Pi_{k-1, l-1}(\vec{x}), \quad 2 \leqslant k \leqslant l \leqslant n .
\end{aligned}
$$

This transformation may be applied in the first instance to the result (4.10) using the same procedure as is used in the proof of Theorem 4.1. This leads to a result of precisely the same character as Theorem 4.10 except that $f_{\gamma}(\vec{x})$ is now replaced by 
$\overline{f_{\gamma}}(\vec{x})$ a homogeneous function of degree $\gamma$ about $(1,0,0, \ldots, 0)$. Moreover, the condition that $f_{\gamma}(\vec{x}) \in H_{\gamma}^{+}$implies only that $\bar{f}_{\gamma}(\vec{x})$ has no singularity, other than the one at $\vec{i}_{1}$ within the closure of $S_{n}$.

Successive applications of this same transformation move the singular point to successive vertices of $S_{n}$, giving the corresponding result for each. This establishes the following generalization of Theorem 4.10 .

THEOREM 5.4. Let $f_{\gamma}^{k}(\vec{x})$ be a homogeneous function of degree $\gamma$ about $\vec{i}_{k}$, this being a specified vertex of $S_{n}$, and let $f_{\gamma}^{k}(\vec{x})$ have no other singularities within the closure of $S_{n}$. Let $\left\{Q\left(\vec{\mu}_{j}\right), j=1,2, \ldots, n !\right\}$ be a quadrature rule set. Then

$$
\begin{aligned}
& Q^{(m)}\left(S_{n}\right) f_{\gamma}^{k}-I\left(S_{n}\right) f_{\gamma}^{k} \\
& \quad \frac{A_{n+\gamma}\left(S_{n} ; Q ; f_{\gamma}^{k}\right)}{m^{n+\gamma}}+\frac{C_{n+\gamma}\left(S_{n} ; Q ; f_{\gamma}^{k}\right) \ln m}{m^{n+\gamma}}+\sum_{s=1} \frac{B_{s}\left(S_{n} ; Q ; f_{\gamma}^{k}\right)}{m^{s}},
\end{aligned}
$$

where the coefficients $B_{s}$ and $C_{n+\gamma}$ satisfy the SSP conditions and $C_{n+\gamma}$ is zero unless $\gamma$ is an integer.

Using this generalization of Theorem 4.10, the theory of the remainder of Section 4 may be extended to cover cases where the singularity is at any specified vertex of $S_{n}$. For example, the result stated involving integrand function (4.18) is equally valid when $r$ given by (4.19) is replaced by $\left|\vec{x}-\overrightarrow{i_{j}}\right|$.

None of the theorems or implied generalizations obtained above are valid when the integrand function has a singularity at more than one vertex. Such functions are excluded by the stated condition that the integrand function and its early derivatives should be integrable except at the vertex under consideration. However, in cases where an integrand function has singularities at two or more vertices of the simplex, the appropriate asymptotic expansion is obtained by concatenating the expansions which are valid for integrand functions having only one vertex singularity. We conclude this paper by outlining the proof of this result, which is in fact an elementary application of Darboux's theorem. Let $f(\vec{x})$ have singularities at some or all of the vertices $\vec{v}^{(k)}$ of the simplex $S_{n}$ but suppose $f(\vec{x})$ can be expanded about each individual vertex $\vec{v}^{(k)}, k=1,2, \ldots, n$, in the form

$$
f(\vec{x})=\sum_{i=0}^{d-1} f_{\alpha_{k}+i}^{(k)}(\vec{x})+g^{(k)}(\vec{x}),
$$

where:

(i) $f_{\gamma}^{(k)}(\vec{x})$ is homogeneous of degree $\gamma$ about $\vec{v}^{(k)}$;

(ii) $g^{(k)}(\vec{x})$ together with all its partial derivatives of total order $l_{k}$ or less are integrable in a region containing $\vec{v}^{(k)}$;

(iii) $g^{(k)}(x)$ is regular within $S_{n}$ but may have integrable singularities at other vertices $\vec{v}^{(j)}, j \neq k$.

We define $G(\vec{x})$ by

$$
f(\vec{x})=\sum_{k=1}^{n} \sum_{i=0}^{d-1} f_{\alpha_{k}+i}^{(k)}(\vec{x})+G(\vec{x})
$$


and examine the nature of $G(\vec{x})$ in a neighborhood of the vertex $\vec{v}^{(j)}$. It follows that

$$
G(\vec{x})=g^{(j)}(\vec{x})-\sum_{\substack{k=1 \\ k \neq j}}^{n} \sum_{i=0}^{d-1} f_{\alpha_{k}+i}^{(k)}(\vec{x}) .
$$

Each term in the double summation is regular at $\vec{v}^{(j)}$. Thus, the nature of the singularity of $G(\vec{x})$ at $\vec{v}^{(j)}$ coincides with the nature of the singularity of $g^{(j)}(\vec{x})$ at $\vec{v}(j)$ which is specified in (ii) above. Since $G(\vec{x})$ is regular, except at the vertices, it follows that $G(\vec{x})$ together with all its partial derivatives of order $l$, with $l=\min _{i} l_{i}$ are integrable over $S_{n}$. Consequently, $G(x)$ is a function to which we can apply Theorem 4.1 with $l=d+n-1$. Since we may apply Theorem 5.4 to each of the individual terms in the double summation in (5.6), we may obtain from (5.6) an expression for $Q^{(m)}\left(S_{n}\right) f-I\left(S_{n}\right) f$. As mentioned above, this turns out to be a concatenation of the individual expansions one would obtain if each singularity were the only one present.

Applied Mathematics Division

Argonne National Laboratory

Argonne, Illinois

Istituto di Calcoli Numerici

University of T urin

Turin, Italy

J. N. LYNESS (1976), "An error functional expansion for $n$-dimensional quadrature with an integrand function singular at the point," Math. Comp., v. 30, pp. 1-23.

J. N. LYNESS (1978), "Quadrature over a simplex: Part 2. A representation for the error functional,” SIAM J. Numer. Anal., v. 15, pp. 870-887. 